Draft version August 7, 2018

Preprint typeset using $\mathrm{LAT}_{\mathrm{E}} \mathrm{X}$ style emulateapj v. 5/2/11

\title{
A SUBSTANTIAL POPULATION OF MASSIVE QUIESCENT GALAXIES AT Z 4 FROM ZFOURGE ${ }^{12}$
}

\author{
Caroline M. S. Straatman ${ }^{1}$, Ivo Labbé ${ }^{1}$, Lee R. Spitler ${ }^{2,11}$, Rebecca Allen ${ }^{3,11}$, Bruno Altieri ${ }^{4}$, Gabriel B. \\ Brammer $^{5}$, Mark Dickinson ${ }^{6}$, Pieter van Dokkum ${ }^{7}$, Hanae Inami ${ }^{6}$, Karl Glazebrook ${ }^{3}$, Glenn G. KaCprzak ${ }^{3,10}$, \\ Lalit KawinwanichakiJ ${ }^{8}$, Daniel D. Kelson ${ }^{9}$, Patrick J. McCarthy ${ }^{9}$, Nicola Mehrtens ${ }^{8}$, Andy Monson ${ }^{9}$, David \\ Murphy $^{9}$, Casey Papovich $^{8}$, S. Eric Persson ${ }^{9}$, Ryan Quadri ${ }^{9}$, Glen Rees ${ }^{2}$, Adam Tomczak ${ }^{8}$, Kim-Vy H. Tran $^{8}$, \\ Vithal Tilvi ${ }^{8}$ \\ Draft version August 7, 2018
}

\begin{abstract}
We report the likely identification of a substantial population of massive $M \sim 10^{11} M_{\odot}$ galaxies at $z \sim 4$ with suppressed star formation rates (SFRs), selected on rest-frame optical to near-IR colors from the FourStar Galaxy Evolution Survey. The observed spectral energy distributions show pronounced breaks, sampled by a set of near-IR medium-bandwidth filters, resulting in tightly con-

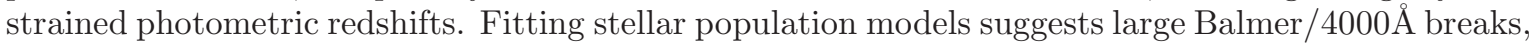
relatively old stellar populations, large stellar masses and low SFRs, with a median specific SFR of $2.9 \pm 1.8 \times 10^{-11} / \mathrm{yr}$. Ultradeep Herschel/PACS $100 \mu \mathrm{m}, 160 \mu \mathrm{m}$ and Spitzer/MIPS $24 \mu \mathrm{m}$ data reveal no dust-obscured SFR activity for 15/19(79\%) galaxies. Two far-IR detected galaxies are obscured QSOs. Stacking the far-IR undetected galaxies yields no detection, consistent with the SED fit, indicating independently that the average specific SFR is at least $10 \times$ smaller than of typical star-forming galaxies at $z \sim 4$. Assuming all far-IR undetected galaxies are indeed quiescent, the volume density is $1.8 \pm 0.7 \times 10^{-5} \mathrm{Mpc}^{-3}$ to a limit of $\log _{10} M / M_{\odot} \geq 10.6$, which is $10 \times$ and $80 \times$ lower than at $z=2$ and $z=0.1$. They comprise a remarkably high fraction $(\sim 35 \%)$ of $z \sim 4$ massive galaxies, suggesting that suppression of star formation was efficient even at very high redshift. Given the average stellar age of $0.8 \mathrm{Gyr}$ and stellar mass of $0.8 \times 10^{11} M_{\odot}$, the galaxies likely started forming stars before $z=5$, with SFRs well in excess of $100 M_{\odot} / y r$, far exceeding that of similarly abundant UV-bright galaxies at $z \geqslant 4$. This suggests that most of the star formation in the progenitors of quiescent $z \sim 4$ galaxies was obscured by dust.
\end{abstract}

Subject headings: galaxies: evolution — galaxies: formation — galaxies: high-redshift — infrared: galaxies - cosmology: observations

\section{INTRODUCTION}

The identification of a population of compact quiescent galaxies at $1<z<3$, characterized by suppressed star formation and very small sizes, has attracted a significant interest (e.g. Daddi et al. 2005; van Dokkum et al. 2008). It remains an open question when these galaxies first appeared. The declining number densities and fractions of quiescent galaxies with redshift at $1<z<3$ suggest they might be rare at $z>3$ (e.g. Muzzin et al.|2013).

\footnotetext{
${ }^{1}$ Leiden Observatory, Leiden University, PO Box 9513, 2300 RA Leiden, The Netherlands; straatman@strw.leidenuniv.nl

${ }^{2}$ Department of Physics and Astronomy, Faculty of Sciences, Macquarie University, Sydney, NSW 2109, Australia

3 Centre for Astrophysics and Supercomputing, Swinburne University, Hawthorn, VIC 3122, Australia

${ }^{4}$ European Space Astronomy Centre (ESAC)/ESA, Villanueva de la Cañada, 28691, Madrid, Spain

${ }^{5}$ European Southern Observatory, Alonso de Córdova 3107, Casilla 19001, Vitacura, Santiago, Chile

6 National Optical Astronomy Observatory, Tucson, AZ, USA

7 Department of Astronomy, Yale University, New Haven, CT 06520, USA

8 George P. and Cynthia W. Mitchell Institute for Fundamental Physics and Astronomy, Department of Physics and Astronomy, Texas A\& M University, College Station, TX 77843

9 Carnegie Observatories, Pasadena, CA 91101, USA

10 Australian Research Council Super Science Fellow

11 Australian Astronomical Observatories, PO Box 915, North Ryde NSW 1670, Australia

12 This paper contains data gathered with the 6.5 meter Magellan Telescopes located at Las Campanas observatory, Chile.
}

Nevertheless, the high ages of some quiescent galaxies at $z \sim 2$ (Whitaker et al. 2013) suggest they could have already existed at much earlier times. If confirmed at $z>3$, massive galaxies must have formed rapidly, early and with an effective mechanism of suppressing star formation.

Beyond $z=3$ candidate early-type or poststarburst galaxies have been reported, despite uncertainties whether their red colors could be due to dustreddening (e.g. Chen \& Marzke 2004; Wiklind et al. 2008: Mancini et al. 2009; Fontana et al. 2009; Guo et al. 2013; Stefanon et al. 2013; Muzzin et al. 2013). In this Letter, we use the FourStar Galaxy Evolution Survey 13 (ZFOURGE; Labbé et al. in preparation) to look for the earliest examples of quiescent galaxies. The strength of ZFOURGE lies in the unique combination of depth and the medium-bandwidth filters (covering $1-2 \mu \mathrm{m}$ ) of the FourStar Infrared Camera (Persson et al. 2013) on the $6.5 \mathrm{~m}$ Magellan Baade Telescope. These enable the derivation of accurate photometric redshifts and the detection of the age-sensitive Balmer/4000 $\AA$ break in faint, red galaxies at $1<z<4.2$.

Throughout, we assume a $\Lambda$ CDM cosmology with $\Omega_{\mathrm{M}}=0.3, \Omega_{\Lambda}=0.7$ and $H_{0}=70 \mathrm{kms}^{-1} \mathrm{Mpc}^{-1}$. The photometric system is $\mathrm{AB}$.

$$
\text { 2. DATA }
$$

13 http://zfourge.tamu.edu 


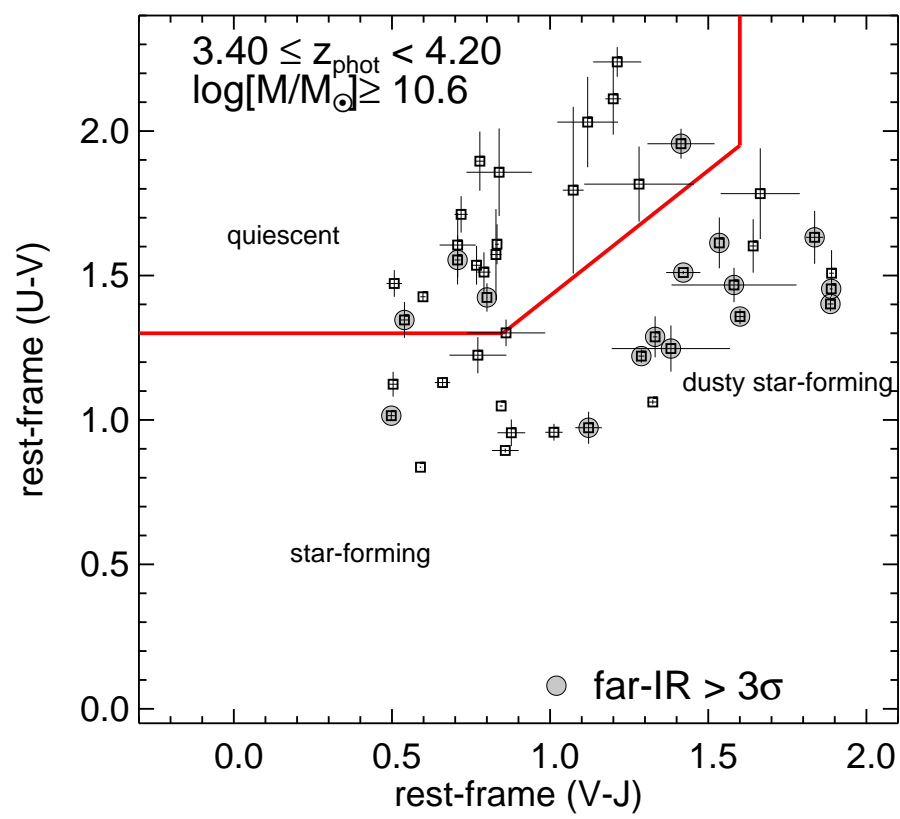

Spitzer/MIPS 24um STACK
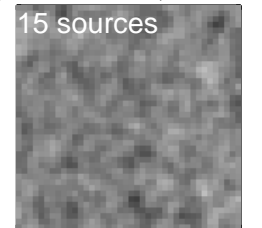

Herschel/Pacs $100 \mu \mathrm{m}$ STACK
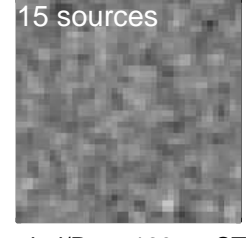

Herschel/Pacs $160 \mu \mathrm{m}$ STACK

15 sources

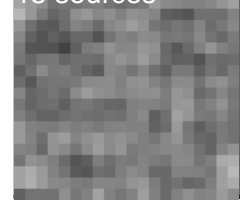

FIG. 1. - Left: Rest-frame UVJ diagram of galaxies with $\log _{10} M / M_{\odot} \geq 10.6$ at $3.4 \leq z<4.2$. The red solid line separates quiescent galaxies (top-left region) from star-forming galaxies. Galaxies with $\geq 3 \sigma$ far-IR detections are indicated with gray filled symbols and tend to be found amongst dusty star-forming galaxies. 19 objects are classified as quiescent, with 15/19 far-IR undetected. Right: $24 \mu \mathrm{m}, 100 \mu \mathrm{m}$ and $160 \mu \mathrm{m}$ stacks $\left(48^{\prime \prime} \times 48^{\prime \prime}\right)$ of undetected quiescent galaxies. Stacking yields no detection: $S_{24 \mu \mathrm{m}}<0.002 \mathrm{mJy}, S_{100 \mu \mathrm{m}}<0.090 \mathrm{mJy}$ and $S_{160 \mu \mathrm{m}}<0.140 \mathrm{mJy}(1 \sigma)$.

ZFOURGE covers three $11^{\prime} \times 11^{\prime}$ pointings in the fields CDFS, COSMOS and UDS, to very deep limits $(\sim 26$ AB total mag $(5 \sigma)$ in $J_{1}, J_{2}, J_{3}$ and $\sim 25 \mathrm{mag}$ in $H_{s}, H_{l}$ and $K_{s}$ ). We combine ZFOURGE with public data, including HUGS (PI:Fontana) HAWK-I and CANDELS (Grogin et al. 2011; Koekemoer et al. 2011) HST/WFC3 imaging, over a wavelength range of $0.3-8 \mu \mathrm{m}$. Full photometric $K_{s}$-band selected catalogs will be presented in Straatman et al. (in preparation).

We use Spitzer/MIPS $24 \mu \mathrm{m}$ data from GOODSSouth (PI: Dickinson), COSMOS (PI: Scoville) an d SPUDS (PI: Dunlop) and ultradeep Herschel/PACS $100 \mu \mathrm{m}$ and $160 \mu \mathrm{m}$ imaging from the GOODS-Herschel (Elbaz et al.2011) and the CANDELS-Herschel program (PI:Dickinson), to independently place constraints on the on-going SFR. The ultradeep PACS $160 \mu \mathrm{m}$ imaging currently provides the best sensitivity for far-IR light from star formation at high redshift, trading off $\mathrm{k}$ correction and source confusion due to increasing beam size (Elbaz et al. 2011), while $24 \mu \mathrm{m}$ data are more sensitive to the presence of hot dust associated with AGN.

Photometric redshifts and rest-frame colors were derived with EAZY (Brammer et al. 2008). Comparing ZFOURGE photometric redshifts to spectroscopic redshifts, Tomczak et al. (2013) found a scatter of $\sigma_{\delta z /(1+z)}=0.019$. Stellar population properties were derived by fitting Bruzual \& Charlot (2003) models with FAST (Kriek et al. 2009), assuming a Chabrier (2003) initial mass function, exponentially declining star formation histories with timescale $\tau$, and solar metallicity.

\section{SELECTION OF QUIESCENT GALAXIES AT $Z \sim 4$.}

We use a two-color criterion (rest-frame $U-V$ versus $V-J$; Figure 1) to separate quiescent galaxies (red in $U-V$, but blue in $V-J$ ) from star-forming galaxies, (blue or red in both $U-V$ and $V-J$ colors) (e.g.,
Labbé et al. 2005; Williams et al. 2009). This technique has been shown to isolate the red sequence of galaxies at $z<3$ (e.g., Whitaker et al. 2011) and was spectroscopically confirmed to identify quiescent galaxies at $z \sim 2$ (e.g. Whitaker et al. 2013).

We focus on the redshift range $3.4 \leq z<4.2$, where the medium-bandwidth filters straddle the Balmer/4000 break. At $z>3.4$ the break enters the $H_{l}$ filter $(1.7 \mu \mathrm{m})$, while at $z<4.2$ the $K_{s}-$ band $(2.2 \mu \mathrm{m})$ still probes light redward of the break. We limit the sample to a signal-to-noise of $>7$ in $K_{s}$ and stellar masses of $\log _{10} M / M_{\odot} \geq 10.6$, where we are complete for passively evolving stellar populations formed at $z<10$. This yields 44 galaxies with high quality photometry, of which 15 fall in the UVJ quiescent region and are undetected in the FIR, a significant fraction: $34 \pm 13 \%$ $(15 / 44)$. A summary of their properties is presented in Table1. Their photometric redshifts range from $z=3.46$ to $z=4.05$ with a mean of $z=3.7$ and mean uncertainty $\delta z /(1+z)=0.036$, leading to well constrained rest-frame colors. Two galaxies have spectroscopic redshifts, with a mean $\left(z_{\text {phot }}-z_{\text {spec }}\right) /\left(1+z_{\text {spec }}\right)=-0.039$ (Szokoly et al. 2004).

\section{PROPERTIES OF QUIESCENT GALAXIES AT $Z \sim 4$}

\subsection{Spectral energy distributions}

We show representative SEDs of seven galaxies in Figure 2. The median SED of all 15 (far-IR undetected) quiescent galaxies, constructed by de-redshifting their photometry and normalising them to the flux density at $4500 \AA$, is also shown.

The observed SEDs are exceedingly faint in the optical ( $I \sim 27$ magnitude) and extremely red throughout the near-IR (median $I-K s=3.7 \pm 0.33$ ). The SEDs are characterized by a sharp break, with $H-K s=1.9 \pm 0.20$ 


\begin{tabular}{|c|c|c|c|c|c|c|c|c|c|c|c|c|c|c|c|}
\hline ID & $\begin{array}{r}\mathrm{ra} \\
(\mathrm{deg}) \\
\end{array}$ & $\begin{array}{r}\text { dec } \\
(\mathrm{deg}) \\
\end{array}$ & $\mathrm{z}_{\mathrm{phot}}$ & U-V & V-J & $\begin{array}{r}\mathrm{M} \\
\left(10^{11} \mathrm{M}_{\odot}\right) \\
\end{array}$ & $\begin{array}{l}\mathrm{SFR}_{\mathrm{SED}} \\
\left(\mathrm{M}_{\odot} / \mathrm{yr}\right) \\
\end{array}$ & 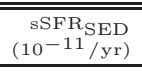 & $\begin{array}{c}\tau^{\mathrm{b}} \\
(\mathrm{Gyr}) \\
\end{array}$ & $\begin{array}{r}\text { age } \\
(\mathrm{Gyr}) \\
\end{array}$ & $\mathrm{A}(\mathrm{V})$ & $\begin{array}{c}\text { Ks_tot }^{\mathrm{C}} \\
(\mathrm{AB})\end{array}$ & $\begin{array}{l}24 \mu \mathrm{m}^{\mathrm{d}} \\
(\mathrm{mJy}) \\
\end{array}$ & $\begin{array}{c}100 \mu \mathrm{m}^{\mathrm{d}} \\
(\mathrm{mJy}) \\
\end{array}$ & $\begin{array}{c}160 \mu \mathrm{m}^{\mathrm{d}} \\
(\mathrm{mJy}) \\
\end{array}$ \\
\hline ZF-CDFS-209 & 53.1132774 & -27.8698730 & $3.56 \pm 0.05$ & $1.43 \pm 0.02$ & $0.60 \pm 0.01$ & 0.76 & 2.239 & 2.884 & 0.10 & 0.63 & 0.3 & 22.6 & $-0.001 \pm 0.004$ & $0.163 \pm 0.163$ & $-0.047 \pm 0.123$ \\
\hline ZF-CDFS-403 & 53.0784111 & -27.8598385 & $3.660^{\mathrm{a}}$ & $1.42 \pm 0.05$ & $0.80 \pm 0.00$ & 1.15 & 31.623 & 27.542 & 0.25 & 0.79 & 0.8 & 22.4 & $0.100 \pm 0.005^{* \dagger}$ & $1.272 \pm 0.199^{*} \dagger \times$ & $\times 1.686 \pm 0.201^{*} \dagger$ \\
\hline ZF-CDFS-617 & 53.1243553 & -27.8516121 & $3.700^{\mathrm{a}}$ & $1.35 \pm 0.06$ & $0.54 \pm 0.01$ & 0.69 & 13.183 & 18.621 & 0.16 & 0.63 & 0.3 & 22.3 & $0.087 \pm 0.003^{* \dagger}$ & $1.062 \pm 0.152^{* \dagger}$ & $0.362 \pm 0.157^{\dagger}$ \\
\hline ZF-CDFS- 4719 & 53.1969414 & -27.7604313 & $3.59 \pm 0.14$ & $1.54 \pm 0.07$ & $0.77 \pm 0.02$ & 0.45 & 0.851 & 1.905 & 0.16 & 1.00 & 0.3 & 23.4 & $-0.000 \pm 0.004$ & $-0.339 \pm 0.175$ & $-0.287 \pm 0.146$ \\
\hline ZF-CDFS- 4907 & 53.1812820 & -27.7564163 & $3.46 \pm 0.16$ & $1.57 \pm 0.16$ & $0.83 \pm 0.02$ & 0.40 & 0.000 & 0.000 & 0.01 & 0.40 & 0.8 & 23.6 & $0.001 \pm 0.004$ & $0.302 \pm 0.132$ & $0.154 \pm 0.107$ \\
\hline ZF-CDFS- 5657 & 53.0106506 & -27.7416019 & $3.56 \pm 0.07$ & $1.61 \pm 0.07$ & $0.83 \pm 0.01$ & 0.76 & 3.311 & 4.467 & 0.25 & 1.26 & 0.3 & & $0.001 \pm 0.005^{\dagger}$ & $0.193 \pm 0.259^{\dagger}$ & $0.078 \pm 0.214^{\dagger}$ \\
\hline ZF-COSMOS- 13129 & 150.1125641 & 2.3765368 & $3.81 \pm 0.17$ & $1.96 \pm 0.05$ & $1.41 \pm 0.11$ & 1.78 & 0.000 & 0.000 & 0.01 & 1.58 & 0.6 & 23.6 & $0.112 \pm 0.010^{*}$ & $0.895 \pm 0.356$ & $-0.215 \pm 0.343$ \\
\hline ZF-COSMOS-13172 & 150.0615082 & 2.3786869 & $3.55 \pm 0.06$ & $1.90 \pm 0.10$ & $0.78 \pm 0.01$ & 1.45 & 0.000 & 0.000 & 0.04 & 0.79 & 0.6 & 22.4 & $0.004 \pm 0.007$ & $-0.007 \pm 0.394$ & $-0.323 \pm 0.420$ \\
\hline ZF-COSMOS- 13414 & 150.0667114 & 2.3823516 & $3.57 \pm 0.19$ & $1.61 \pm 0.11$ & $0.71 \pm 0.06$ & 0.44 & 0.035 & 0.079 & 0.10 & 1.00 & 0. & 23.4 & $0.009 \pm 0.008$ & $-0.330 \pm 0.546$ & $0.194 \pm 0.452$ \\
\hline ZF-UDS- 885 & 34.3685074 & -5.2994704 & $3.99 \pm 0.41$ & $1.80 \pm 0.29$ & $1.07 \pm 0.03$ & & 0.001 & 0.001 & 0.03 & 0.40 & 1. & 24.0 & $0.012 \pm 0.008$ & $1.009 \pm 0.537$ & $-0.089 \pm 0.400$ \\
\hline ZF-UDS- 1236 & 34.3448868 & -5.2925615 & $3.58 \pm 0.08$ & $1.47 \pm 0.05$ & $0.51 \pm 0.02$ & 0.60 & 0.550 & 0.912 & 0.06 & 0.50 & 0.4 & 22.6 & $-0.016 \pm 0.011$ & $0.383 \pm 0.449$ & $0.537 \pm 0.360$ \\
\hline ZF-UDS- 2622 & 34.2894516 & -5.2698011 & $3.77 \pm 0.10$ & $1.51 \pm 0.07$ & $0.79 \pm 0.02$ & 0.87 & 16.218 & 18.621 & 0.16 & 0.63 & 0.9 & 23.0 & $0.013 \pm 0.010$ & $0.761 \pm 0.372$ & $0.152 \pm 0.442$ \\
\hline ZF-UDS-3112 & 34.2904282 & -5.2620673 & $3.53 \pm 0.06$ & $1.71 \pm 0.06$ & $0.72 \pm 0.02$ & 0.43 & 1.862 & 4.467 & 0.25 & 1.26 & 0. & 23.2 & $-0.010 \pm 0.010$ & $-0.547 \pm 0.377$ & $0.256 \pm 0.333$ \\
\hline ZF-UDS-5418 & 34.2937546 & -5.2269468 & $3.53 \pm 0.07$ & $1.55 \pm 0.08$ & $0.71 \pm 0.01$ & 0.44 & 3.020 & 6.761 & 0.16 & 0.79 & 0. & 23.3 & $0.049 \pm 0.010^{*}$ & $0.560 \pm 0.443$ & $-0.494 \pm 0.386$ \\
\hline ZF-UDS-6119 & 34.2805405 & -5.2171388 & $4.05 \pm 0.27$ & $1.86 \pm 0.15$ & $0.84 \pm 0.10$ & 0.55 & 5.623 & 10.471 & 0.10 & 0.50 & 1. & 23.8 & $-0.013 \pm 0.009$ & $0.224 \pm 0.477$ & $0.331 \pm 0.316$ \\
\hline ZF-UDS-9526 & 34.3381844 & -5.1661916 & $3.97 \pm 0.18$ & $2.11 \pm 0.12$ & $1.20 \pm 0.03$ & 0.89 & 16.596 & 18.621 & 0.16 & 0.63 & 1.8 & 24.2 & $0.016 \pm 0.009$ & $0.038 \pm 0.351$ & $-0.000 \pm 0.294$ \\
\hline ZF-UDS-10401 & 34.3601379 & -5.1530914 & $3.91 \pm 0.38$ & $1.82 \pm 0.13$ & $1.28 \pm 0.17$ & 0.38 & 0.001 & 0.002 & 0.02 & 0.25 & 1.7 & 24.6 & $0.007 \pm 0.010$ & $-0.500 \pm 0.340$ & $-0.490 \pm 0.383$ \\
\hline ZF-UDS- 10684 & 34.3650742 & -5.1488328 & $3.95 \pm 0.48$ & $2.03 \pm 0.16$ & $1.12 \pm 0.10$ & 0.85 & 3.802 & 4.467 & 0.25 & 1.26 & 1.0 & 24.1 & $0.007 \pm 0.012$ & $-0.105 \pm 0.388$ & $-0.503 \pm 0.544$ \\
\hline ZF-UDS- 11483 & 34.3996315 & -5.1363320 & $3.63 \pm 0.32$ & $2.24 \pm 0.05$ & $1.21 \pm 0.08$ & 1.02 & 0.000 & 0.000 & 0.01 & 1.00 & 1.0 & 23.6 & $0.004 \pm 0.011$ & $-0.149 \pm 0.409$ & $0.715 \pm 0.325$ \\
\hline
\end{tabular}

${ }^{\text {a }}$ Spectroscopic redshift from Szokoly et al. (2004).

b $\mathrm{SFR} \sim \exp (-\mathrm{t} / \tau)$.

c Total magnitude in the FourStar $K_{s}$-band.

d “*”: $\geq 3 \sigma$ detections. "†": X-ray detected (Szokoly et al. 2004; Xue et al., 2011). "× ": radio source (Miller et al., 2013). 

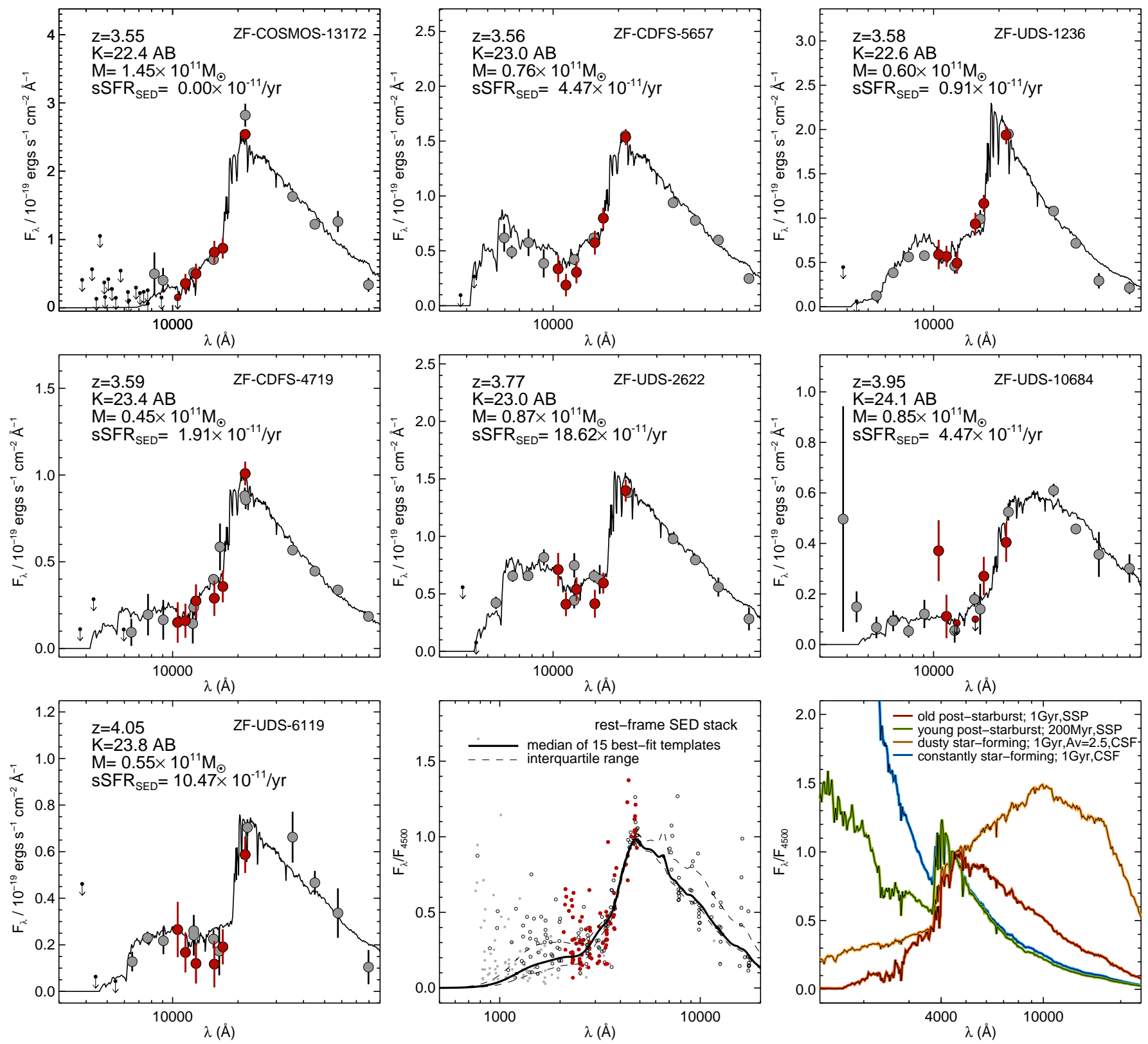

FIG. 2.- Observed SEDs of UVJ selected quiescent galaxies. Red datapoints correspond to the FourStar medium-bandwidth filters. The solid curve is the fitted model from FAST. Downward pointing arrows are $1 \sigma$ upper limits. Bottom-middle: Rest-frame SED of the 15 far-IR undetected galaxies (open symbols), normalized at rest-frame $4500 \AA$, with gray symbols corresponding to $1 \sigma$ upper limits. The solid curve is the median of the best-fit template SEDs. Dashed lines mark the interquartile range. Bottom-right: Four model SEDs with constant star formation or a single stellar population (SSP) and ages from 200Myr to 1Gyr. The observed SEDs are characterized by pronounced Balmer/4000Å breaks, similar to the old post-starburst model. 
and peaking in $K_{s}$, and a blue spectral slope in the midIR Spitzer/IRAC bands $(K s-[4.5 \mu \mathrm{m}]=0.91 \pm 0.13)$. The break is reminiscent of the strong break found in quiescent galaxies at lower redshift, where it is caused by combination of the Balmer and $4000 \AA$ absorption features, indicative of a combination of relatively old stellar populations and suppressed star formation. Additionally, some galaxies exhibit a second break at bluer wavelengths, which is likely the Lyman break.

As it is difficult to separate the contribution of the Balmer break and the $4000 \AA$ break from photometry alone, we quantify the size of the total combined break $\left(D_{\text {tot }}\right)$ by estimating the flux $\left(F_{\nu}\right)$ ratio at $4000-$ $4100 \AA$ and $3500-3650 \AA$ on the best-fit models of each galaxy individually, extending the definition of D4000n (Balogh et al. 1999) to cover the Balmer break as well. We find the median $D_{t o t}=2.8 \pm 0.1$, which is in the range of post-starburst galaxies with suppressed star formation (e.g., a $\tau=10$ Myr model produces $D_{t o t}=3.1$ at $500 \mathrm{Myr})$. In contrast, unobscured constant star-forming (CSF) models only reach $D_{\text {tot }}=1.8$ at $1 \mathrm{Gyr}$. Heavily obscured star-forming models (e.g. $1 \mathrm{Gyr}, \mathrm{CSF}, A_{v}=2.5$ ) can also reach quite red $D_{\text {tot }}=2.5$, but are ruled out as they predict very red $K s-[4.5 \mu \mathrm{m}]=2.0$, whereas the observed SED-slopes are bluer.

\subsection{Stellar population fits}

Models with exponentially declining SFRs fit the data well, with a median $\chi_{\text {red }}^{2}=1.3$. The median bestfit age of the galaxies is $0.8 \mathrm{Gyr}$, the median star formation timescale $(\tau) 0.1 \mathrm{Gyr}$, the average stellar mass $0.8 \times 10^{11} M_{\odot}$, and the median specific $\mathrm{SFR}(\mathrm{sSFR})$ $2.9 \pm 1.8 \times 10^{-11} / \mathrm{yr}$.

To test if models with ongoing star formation provide acceptable fits to the sample, we force $\tau=250 \mathrm{Myr}$, $\tau=1 \mathrm{Gyr}$ or CSF and refitted the data, finding a median $\chi_{\text {red,250Myr }}^{2}=2.1, \chi_{\text {red, } 1 \text { Gyr }}^{2}=6.6$ and $\chi_{\text {red,CSF }}^{2}=6.6$. This shows that models with $\tau=250 \mathrm{Myr}$ provide almost equally good fits, but longer formation timescales $(\tau>1 \mathrm{Gyr})$ provide poor fits to the data. For all individual galaxies $\tau<250 \mathrm{Myr}$ models produced better fits than did $\tau>1$ Gyr models. We note that high redshift solutions with low sSFRs are preferred in all cases. Other solutions, e.g. at low redshift or with ongoing obscured star formation are ruled out at more than $99 \%$ confidence for 18/19 galaxies.

We refitted the data using the models of Maraston (2005), and obtained a mean stellar mass of $0.5 \times 10^{11} M_{\odot}$, with a typical offset of -0.2 dex compared to the masses in Table 1, and a median sSFR of $0.1 \times 10^{-11} / \mathrm{yr}$. Hence the result is not strongly dependent on the adopted stellar population model.

Overall, the fits suggest most stars were formed at $z>5$, followed by an epoch of suppressed star formation. As expected, the median stellar ages are lower than the typical age of 1.3 Gyr found at $z \sim 2$ by Whitaker et al. (2013). We find some galaxies with very red $U-V$ and $V-J$, pointing towards older stellar populations. However, their best-fit ages are the same as for the bluer galaxies, with larger redshift uncertainties or dust, suggesting that dust and photometric scatter are the main causes.

\subsection{Independent constraints on SFR and AGN activity from Herschel}

We derived Spitzer/MIPS $24 \mu \mathrm{m}$, Herschel/PACS $100 \mu \mathrm{m}$ and $160 \mu \mathrm{m}$ flux intensities, measured in apertures of $7^{\prime \prime}, 8^{\prime \prime}$ and $12^{\prime \prime}$ diameter with aperture corrections of $2.56,2.45$ and 2.60 , respectively (assuming a point source profile). Light from neighbouring sources was subtraced following Labbé et al. (2010).

We find 1,2 and $4>3 \sigma$ detections at $160 \mu \mathrm{m}, 100 \mu \mathrm{m}$ and $24 \mu \mathrm{m}$, respectively. These $4 / 19$ galaxies may have obscured star formation. The total detection rate $(21 \pm$ $11 \%$ ) is lower than the $50 \%$ of $24 \mu \mathrm{m}$ detections reported earlier for $z>3$ quiescent galaxies (Stefanon et al. 2013). Two are also detected in X-ray and are type-2 QSOs (Szokoly et al. 2004; Xue et al. 2011), of which one is a radio source (Miller et al. 2013). A third X-ray detection is found amongst the far-IR undetected galaxies, for a total of 3 likely AGN.

To place tighter constraints on the average far-IR luminosity of the 15 far-IR undetected galaxies, we stack their $24 \mu \mathrm{m}, 100 \mu \mathrm{m}$ and $160 \mu \mathrm{m}$ images (Figure 1), with uncertainties derived by bootstrap resampling. The formal measurements are $S_{24} \mu \mathrm{m}=0.001 \pm 0.002 \mathrm{mJy}, S_{100} \mu \mathrm{m}=$ $0.049 \pm 0.090 \mathrm{mJy}$ and $S_{160 \mu \mathrm{m}}=0.039 \pm 0.140 \mathrm{mJy}$. Hence the sources are undetected. The strongest constraint on obscured SFR is obtained at $160 \mu \mathrm{m}$. Using the IR templates of Wuyts et al. (2011), we find $7.1 \pm 25 M_{\odot} / \mathrm{yr}$. Given the mean mass of the sample $\left(0.8 \times 10^{11} M_{\odot}\right)$, this corresponds to a sSFR of $0.9 \pm 3 \times 10^{-10} \mathrm{yr}^{-1}$. While these independently derived limits cannot rule out ongoing obscured star formation, they are consistent with the SED fits $\left(2.9 \pm 1.8 \times 10^{-11} / \mathrm{yr}\right)$, and are $\sim 10 \times$ smaller than the $\mathrm{sSFR}=3-6 \times 10^{-9} / \mathrm{yr}$ of similarly massive galaxies at $z \sim 3$ and typical UV-bright star-forming galaxies at $z \sim 4$ (e.g. Stark et al. 2013; Viero et al. 2013).

\subsection{Contamination by emission lines}

We caution that the galaxies here could in fact be vigorously star-forming, if their $K_{s}$-band fluxes were boosted dramatically by emission lines ([OIII] and $H \beta$ ), mimicking a Balmer Break (e.g. Shim et al. 2011; Stark et al. 2013). We tested this scenario by fitting CSF models to the SEDs without fitting the $K_{s}$-band, leading to fits with high obscuration (median $A(V) \sim 2$ ).

The CSF models fit the data poorly and vastly underpredict the median $K_{s}$-band: $\left(K_{s, o b s}-K_{s, S E D}\right)_{C S F}=$ $-0.53 \pm 0.06$ (note that standard $-\tau$ models predict the $K_{s}$-band magnitude nearly perfectly: $\left(K_{s, o b s}-\right.$ $\left.\left.K_{s, S E D}\right)_{\tau=\text { free }}=-0.01 \pm 0.05\right)$. Assuming this excess is due to strong $\left(E W_{\text {obs }} \sim 2000 \AA\right)$ emission lines, the predicted median SFR is $\sim 1000 M_{\odot} / \mathrm{yr}$, which, because of the high obscuration, should result in $7-18 \sigma$ detections in $160 \mu \mathrm{m}$, but is not observed.

Furthermore, we can test the hypothesis that $K_{s}$ is boosted by $[O I I I](\lambda \lambda 4959,5007 \AA)$ and $H \beta(4861 \AA)$ at $3.0<z<3.6$, by looking at existing narrowband $N B 209(2.10 \mu \mathrm{m})$ data, covering CDFS+COSMOS (Lee et al. 2012). Since the odds are only $20 \%$ that $N B 209$ is affected by any of these lines, it effectively traces the continuum. Using a simple model, drawing uniformly random redshifts at $3.0<z<3.6$, the predicted median color is $\left(K_{s}-N B 209\right)=-0.44$, nearly independent of line ratios, in strong disagreement with the 

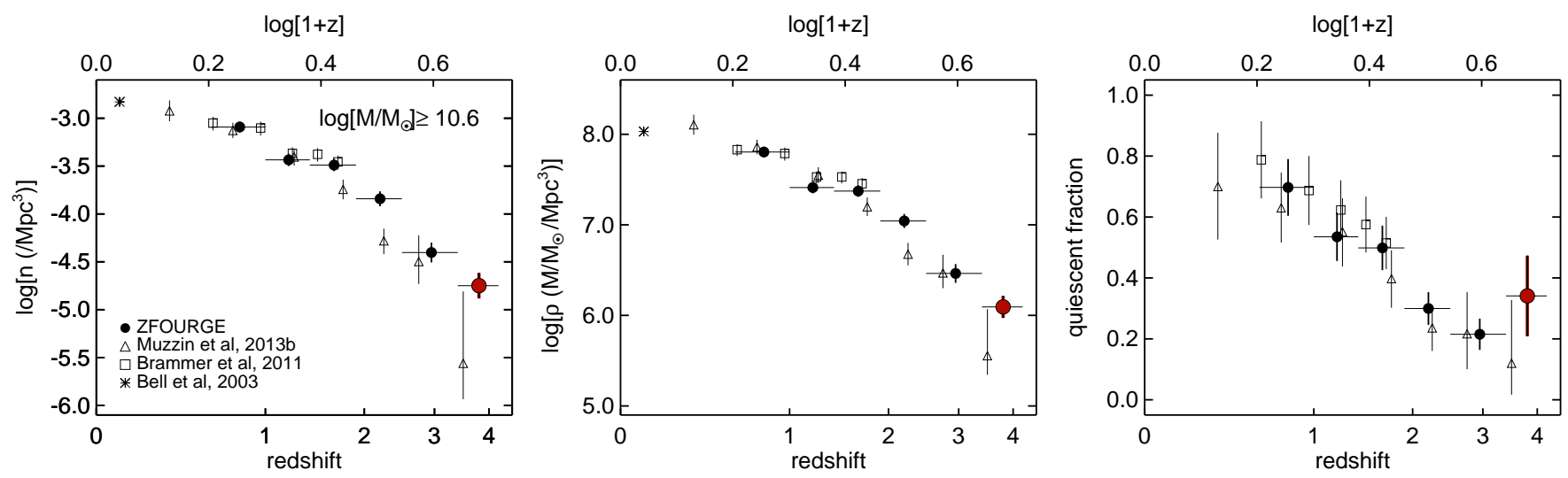

FIG. 3.- Left: Number density of quiescent galaxies in ZFOURGE. Middle: Stellar mass density. Right: Quiescent fraction. Errors are a combination of the Poisson uncertainty and cosmic variance. Horizontal errorbars indicate the width of the redshift bins. The red symbols denote the 15 UVJ selected and far-IR undetected galaxies. We compare with Bell et al. (2003) at $z=0.1$, Brammer et al. (2011) to $z<2$ and Muzzin et al. (2013) at $0<z<4$. The overall trend is a decrease in number density towards $z \sim 4$, consistent with the earlier NMBS and UltraVISTA results. However, the larger depth and sampling of ZFOURGE allows for much better constraints on the evolution at $2<z<4$. Surprisingly, at $z=3.7,34 \pm 13 \%$ of the galaxies with $\log _{10} M / M_{\odot} \geq 10.6$ could be quiescent, suggesting that the decline of the quiescent fraction could flatten at $z \gtrsim 2-3$.

observed median $\left(K_{s}-N B 209\right)=-0.04 \pm 0.1$. We also inspected data from the 3D-HST survey (Brammer et al. 2012), with low resolution spectral coverage at $1.1-$ $1.6 \mu \mathrm{m}$, as strong $[O I I I] / H \beta$ lines in $K_{s}$ would imply strong $[\mathrm{OII}]$ in the HST/WFC3 grism. We found only 2 detections for 13 galaxies with coverage to an emission line sensitivity of $\sim 5 \times 10^{-17} \mathrm{ergs} / \mathrm{s} / \mathrm{cm}^{2}(5 \sigma)$ : $M g I I(\lambda 2798 \AA)$ for the QSO ZF-CDFS-617 and [OII] for the $24 \mu \mathrm{m}$ detected ZF-UDS-5418.

\section{IMPLICATIONS}

From hereon we adopt as operational definition of "quiescent": galaxies that satisfy the UVJ criterium and are not detected in the far-IR (e.g. Bell et al. 2012). We note however, that the current data do not allow to determine conclusively whether the galaxies have completely stopped forming stars as the sample is too faint for spectrographs on large telescopes.

\subsection{Number densities}

From the 15 quiescent galaxies we estimate the volume and stellar mass density, finding $1.8 \pm 0.7 \times 10^{-5} \mathrm{Mpc}^{-3}$ and $1.2 \pm 0.5 \times 10^{6} \mathrm{M}_{\odot} \mathrm{Mpc}^{-3}$, respectively. Uncertainties are the quadratic sum of the Poission uncertainty and variations due to large scale structure (Moster et al. 2011). The volume density in the $11^{\prime} \times 11^{\prime}$ area in the ZFOURGE-UDS field is $\sim 3 \times$ higher than in the ZFOURGE-COSMOS field, underscoring the need for probing mulitple pointings to faint limits.

For comparison with other surveys, we integrated the COSMOS/UltraVISTA mass function at $3<z<4$ for quiescent galaxies of Muzzin et al. (2013), based on a similar UVJ classification, to $\log _{10} M / M_{\odot} \geq 10.6$. UltraVISTA produces a number density of $2.7 \times 10^{-6} \mathrm{Mpc}^{-3}$ and a mass density of $3.1 \times 10^{5} \mathrm{M}_{\odot} \mathrm{Mpc}^{-3}$. These are factors of $\sim 7$ and $\sim 4$, lower (albeit at only $\sim 1.6 \sigma$ significance). This is likely a completeness effect, as UltraVISTA is only complete to $M \gtrsim 10^{11} M_{\odot}$. Indeed, Muzzin et al. (2013) select galaxies with $K_{s, t o t, A B}<$ 23.4 , while $50 \%$ of the galaxies here have $K_{s, t o t, A B}>$ 23.4 .
The number and stellar mass densities of quiescent galaxies at $0.6 \leq z<4.2$ are shown in Figure 3 These were obtained from the full ZFOURGE catalogs (Straatman et al., in prep), using the same selection criteria as described in section 3 . The number density decreases rapidly towards $z \sim 4(\sim 10 \times$ lower than at $z=2$ and $\sim 80 \times$ than at $z=0.1$ ), suggesting that a small fraction $(10-15 \%)$ of $z \sim 2$ quiescent galaxies was already in place at $z \sim 4$. The last panel of Figure 3 shows the fraction of quiescent galaxies with $\log _{10} M / M_{\odot} \geq 10.6$. This strongly declines with redshift between $0.6<z<3$. Therefore, we would expect a value close to zero at $z \sim 4$, but we find a surprisingly high fraction of $34 \pm 13 \%$. This is similar to the value at $z \sim 2.2(30 \pm 8 \%)$, suggesting a flat quiescent fraction at $2<z<4$.

\subsection{Star-forming progenitors}

Given average stellar ages of $0.8 \mathrm{Gyr}$ and masses of $0.8 \times 10^{11} M_{\odot}$, the galaxies likely started forming their stars much earlier than $z=5$, with SFRs well in excess of $100 M_{\odot} / y r$. This raises the question what are the likely progenitors. In recent years, UV-luminous galaxies have been found in large numbers to $z \sim 10$ (e.g. Bouwens et al. 2013; Ellis et al. 2013). These are actively star-forming, although even the most luminous galaxies found so far at $z>4$ have relatively modest UV-derived SFRs $\left(<100 M_{\odot} /\right.$ yr $)$ (Smit et al. 2012).

The expected number density at $z>4$ of the progenitors depends on the assumed star formation timescale (gray shaded area in Figure 44). If the progenitors were visible at all times (i.e. a formation timescale of $\sim 1 \mathrm{Gyr}$ and number density of $\left.1.8 \pm 0.7 \times 10^{-5} \mathrm{Mpc}^{-3}\right)$, then we can use the SFR functions at $z=4-7$ (Smit et al. 2012) to select progenitors with sufficiently high SFRs on fixed cumulative number density (van Dokkum et al. 2010).

As shown in Figure 4 this number density falls $~ 1.2$ dex short. If we assume shorter formation timescales (e.g. a few 100Myr), the progenitors require much higher SFRs and are predicted to be found in smaller numbers. Comparing to UV-luminosity functions from widefield surveys, using the redshift window as the formation 


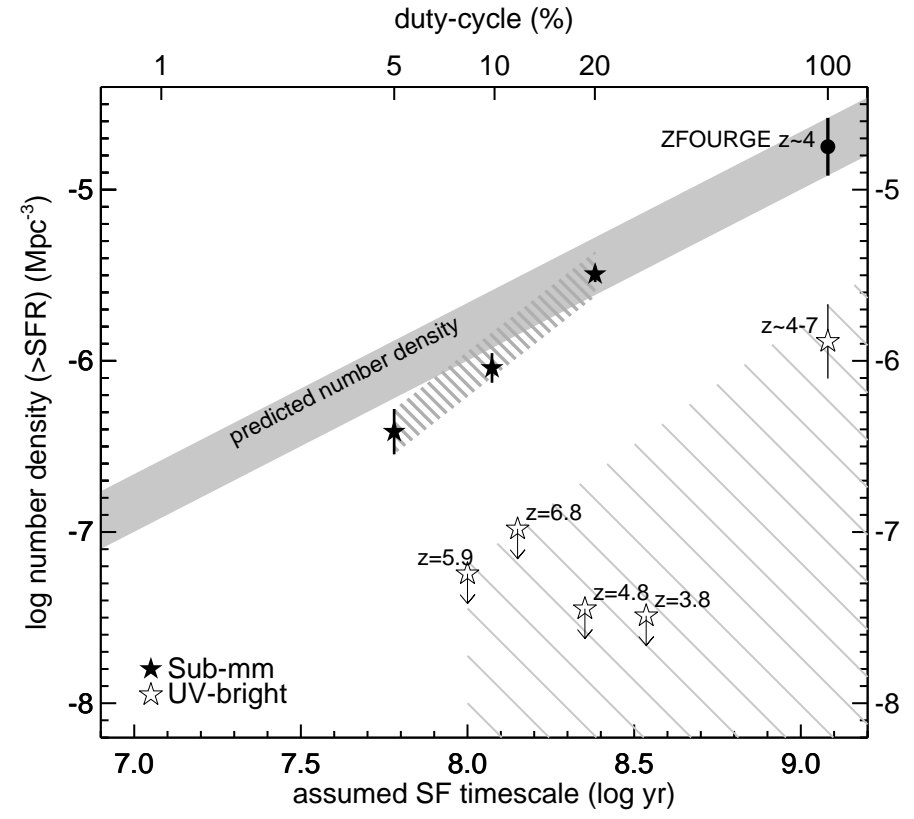

FIG. 4.- The predicted cumulative number density $\left(\mathrm{n}(>\mathrm{SFR}) / \mathrm{Mpc}^{3}\right)$ for the progenitors of the $z \sim 4$ quiescent galaxies as a function of the assumed formation timescale. The gray filled area shows the expected range in number density based on the $z \sim 4$ sample. If the galaxies form over a long timescale (e.g. $\sim 1$ Gyr), this is simply the observed number density. If they form in shorter, more intense bursts (smaller duty-cycles), the observed progenitor number densities are expected to be smaller. Open star symbols are estimates from the SFR functions of Smit et al. (2012) $(z \sim 4-7)$ and the UV-luminosity functions of van der Burg et al. (2010) $(z=3.8$ and $z=4.8)$, Willott et al. (2013) $(z=5.9)$ and Bowler et al. (2012) $(z=6.8)$. For the latter we assume that $\log _{10} \mathrm{SFR}=-0.4\left(M_{1600}-\left\langle A_{1600}\right\rangle\right)-7.25$ (Kennicutt 1998), with $M_{1600}$ the luminosity at $1600 \AA$ in AB mag and $\left\langle A_{1600}\right\rangle$ the dust-correction factor from Bouwens et al. (2012). Upper limits are derived from the respective survey volumes. Filled stars show sub-mm number counts (Karim et al. 2013) using a conversion of $1 \mathrm{mJy} \approx 1.667 \times 10^{12} L_{\odot, I R}$ (Blain et al. 2002) and $1 M_{\odot} / y r=4.5 \times 10^{-44} L_{I R}($ ergs $/ s)$ (Kennicutt 1998) and assuming $10 \%$ of these are at $z>4$. The number of luminous UV-bright galaxies at $z>4$ is far too low (1-2 dex), while the sub-mm counts are much better matched, suggesting there might be sufficient numbers of heavily obscured starburst galaxies at $z>4$ if their formation timescales are $\sim 200 \mathrm{Myr}$.

timescale, the number densities are $>1.5$ dex too low, reflecting that sufficiently luminous UV-bright galaxies are extremely rare.

Alternatively, the main star formation episode is obscured by dust. There exists a population of high-redshift sub-mm detected galaxies, including highly obscured gas-rich mergers (e.g. Younger et al. 2007), with large SFRs $\left(\gtrsim 1000 M_{\odot} / \mathrm{yr}\right)$, that could be progenitors of $z \sim 4$ quiescent galaxies. Based on the $870 \mu \mathrm{m}$ source counts of Karim et al. (2013), and tentatively assuming that 10\% are at $z>4$ (e.g. Swinbank et al. 2012), we find that obscured starbursting galaxies are sufficiently numerous. This suggests that most of the star formation in the progenitors of quiescent $z \sim 4$ galaxies could have been obscured by dust.

\section{SUMMARY}

Using very deep imaging from ZFOURGE we find evidence for the existence of massive $\left(M \sim 10^{11} M_{\odot}\right)$ galaxies with suppressed star formation at very early times $(z \sim 4)$. The galaxies satisfy the UVJ criterium, which has been shown to efficiently select quiescent galaxies at $z<3$ (Whitaker et al. 2011, 2013; ; Williams et al. 2009). The observed SEDs show prominent breaks, well sampled by the FourStar near-IR medium-bands, leading to accurate photometric redshifts and illustrating a key strength of the survey. The SEDs are well fit by models with strong Balmer $/ 4000 \AA$ breaks, small $\tau(<250 \mathrm{Myr})$, high ages $(\sim 0.8 \mathrm{Gyr})$ and low sSFRs $\left(2.9 \pm 1.8 \times 10^{-11} / \mathrm{yr}\right)$. Consistent with this, $79 \%$ of the galaxies are undetected in deep Spitzer/MIPS and Herschel/PACS imaging. Stacking the far-IR places an independent constraint on the average sSFR of $<3 \times 10^{-10} \mathrm{yr}^{-1}$, a factor $>10 \times$ smaller than the average sSFR of UV-bright star-forming galaxies at these redshifts (e.g. Stark et al. 2013) and consistent with these galaxies having strongly suppressed SFRs.

While rare (with number densities $\sim 10 \times$ and $\sim 80 \times$ lower than at $z=2$ and $z=0.1$ ), they make up a surprisingly high fraction of the massive galaxy population at $z \sim 4(34 \pm 13 \%)$, higher than expected based on the declining trend over $1<z<3$, suggesting an effective mechanism of suppressing star formation and short formation timescales $(<1 \mathrm{Gyr})$. The implied SFRs needed to form galaxies with a mean stellar mass of $0.8 \times 10^{11} M_{\odot}$ in such a short time exceeds that of similarly abundant UV-bright galaxies at $z \geqslant 4$, suggesting that most of the star formation in their progenitors was obscured by dust.

We emphasize that without spectroscopic confirmation the number of quiescent galaxies at $z \sim 4$ remains poorly constrained, but given their faint magnitudes, real progress will likely have to wait until the launch of JWST or construction of ELTs. Currently, ALMA observations can place stronger limits on the dust-obscured activity of these galaxies and help identify the progenitors at $z \gtrsim 4$.

\section{ACKNOWLEDGEMENTS}

This research was supported in part by the George P. and Cynthia Woods Mitchell Institute for Fundamental Physics and Astronomy. We would especially like to thank the Mitchell family for their continuing support. We thank Danilo Marchesini and Adam Muzzin for help with the UltraVISTA number densities. We thank Marijn Franx, Mattia Fumagalli, Shannon Patel, Jesse van de Sande, David Sobral, Renske Smit, Paul van der Werf and Claudia Maraston for useful discussions. We acknowledge support by the following grants: NSF AST1009707, ERC HIGHZ \#227749 and NL-NWO Spinoza. Australian access to the Magellan Telescopes was supported through the NCRIS of the Australian Federal Government. This work is based on observations made with Herschel, an ESA Cornerstone Mission with significant participation by NASA, through an award issued by JPL/Caltech. 


\section{REFERENCES}

Balogh, M. L., Morris, S. L., Yee, H. K. C., Carlberg, R. G., \& Ellingson, E. 1999, ApJ, 527, 54

Bell, E. F., McIntosh, D. H., Katz, N., \& Weinberg, M. D. 2003, ApJS, 149, 289

Bell, E. F., et al. 2012, ApJ, 753, 167

Blain, A. W., Smail, I., Ivison, R. J., Kneib, J.-P., \& Frayer, D. T. 2002, Phys. Rep., 369, 111

Bouwens, R. J., et al. 2012, ApJ, 754, 83

-. 2013, ApJ, 765, L16

Bowler, R. A. A., et al. 2012, MNRAS, 426, 2772

Brammer, G. B., van Dokkum, P. G., \& Coppi, P. 2008, ApJ, 686,1503

Brammer, G. B., et al. 2011, ApJ, 739, 24

-. 2012, ApJS, 200, 13

Bruzual, G., \& Charlot, S. 2003, MNRAS, 344, 1000

Chabrier, G. 2003, PASP, 115, 763

Chen, H.-W., \& Marzke, R. O. 2004, ApJ, 615, 603

Daddi, E., et al. 2005, ApJ, 626, 680

Elbaz, D., et al. 2011, A\&A, 533, A119

Ellis, R. S., et al. 2013, ApJ, 763, L7

Fontana, A., et al. 2009, A\&A, 501, 15

Grogin, N. A., et al. 2011, ApJS, 197, 35

Guo, Y., et al. 2013, ApJS, 207, 24

Karim, A., et al. 2013, MNRAS, 432, 2

Kennicutt, Jr., R. C. 1998, ARA\&A, 36, 189

Koekemoer, A. M., et al. 2011, ApJS, 197, 36

Kriek, M., van Dokkum, P. G., Labbé, I., Franx, M., Illingworth, G. D., Marchesini, D., \& Quadri, R. F. 2009, ApJ, 700, 221

Labbé, I., et al. 2005, ApJ, 624, L81

-. 2010, ApJ, 716, L103

Lee, J. C., et al. 2012, PASP, 124, 782

Mancini, C., Matute, I., Cimatti, A., Daddi, E., Dickinson, M., Rodighiero, G., Bolzonella, M., \& Pozzetti, L. 2009, A\&A, 500, 705

Maraston, C. 2005, MNRAS, 362, 799
Miller, N. A., et al. 2013, ApJS, 205, 13

Moster, B. P., Somerville, R. S., Newman, J. A., \& Rix, H.-W. 2011, ApJ, 731, 113

Muzzin, A., et al. 2013, ApJ, 777, 18

Persson, S. E., et al. 2013, PASP, 125, 654

Shim, H., Chary, R.-R., Dickinson, M., Lin, L., Spinrad, H., Stern, D., \& Yan, C.-H. 2011, ApJ, 738, 69

Smit, R., Bouwens, R. J., Franx, M., Illingworth, G. D., Labbé, I., Oesch, P. A., \& van Dokkum, P. G. 2012, ApJ, 756, 14

Stark, D. P., Schenker, M. A., Ellis, R., Robertson, B., McLure, R., \& Dunlop, J. 2013, ApJ, 763, 129

Stefanon, M., Marchesini, D., Rudnick, G. H., Brammer, G. B., \& Whitaker, K. E. 2013, ApJ, 768, 92

Swinbank, A. M., et al. 2012, MNRAS, 427, 1066

Szokoly, G. P., et al. 2004, ApJS, 155, 271

Tomczak, A. R., et al. 2013, ArXiv e-prints

van der Burg, R. F. J., Hildebrandt, H., \& Erben, T. 2010, A\&A, 523, A74

van Dokkum, P. G., et al. 2008, ApJ, 677, L5

-. 2010, ApJ, 709, 1018

Viero, M. P. et al. 2013, ApJ, 779, 32

Whitaker, K. E., et al. 2011, ApJ, 735, 86

-. 2013, ApJ, 770, L39

Wiklind, T., Dickinson, M., Ferguson, H. C., Giavalisco, M. Mobasher, B., Grogin, N. A., \& Panagia, N. 2008, ApJ, 676, 781

Williams, R. J., Quadri, R. F., Franx, M., van Dokkum, P., \& Labbé, I. 2009, ApJ, 691, 1879

Willott, C. J., et al. 2013, AJ, 145, 4

Wuyts, S., et al. 2011, ApJ, 738, 106

Xue, Y. Q., et al. 2011, ApJS, 195, 10

Younger, J. D., et al. 2007, ApJ, 671, 1531 